\title{
Investigation of Locally Resolved Current Density Distribution of Segmented PEM Fuel Cells to Detect Malfunctions
}

\author{
R. Lin ${ }^{\mathrm{a}, \mathrm{b}}$, H. Sander ${ }^{\mathrm{a}}$, E. Gülzow ${ }^{\mathrm{a}}$, A. K. Friedrich ${ }^{\mathrm{a}}$ \\ a Institute of Technical Thermodynamics, German Aerospace Center (DLR), Stuttgart, \\ 70569 Germany \\ b School of Automotive Studies, Tongji University, Shanghai, 201804 China
}

\begin{abstract}
With the aid of PCB technology, current density distribution could be achieved by measuring the local current density inside the fuel cell, which would be more precisely reflecting the local electrochemical reaction. An important application of segmented cell technology was used for in-situ error detection of the evolution of membrane leakage. From the evolution of current density distributions, starting locations of membrane leakages and their spreading could be detected. The effect of operation conditions could be found: the steeply decreased pressure and the fast cooling of the local membrane was the main reason for the degradation of the membrane. It caused large temperature gradient in different local membranes, which resulted in inhomogeneous current density distribution and mechanical strain and stress. With the aid of segmented cell, malfunction could be detected at an early stage and thereby catastrophic failure of the whole stack may be avoided.
\end{abstract}

\section{Introduction}

In order to achieve a better understanding of the electrochemical processes in polymer electrolyte fuel cells (PEMFC) and to investigate effects of operating conditions on the local performance a variety of locally resolved current density distribution mapping techniques have been developed (1-3) . Printed circuit boards (PCB) with segments and internal measurement resistances have been developed to measure current density distributions in fuel cells both in single cells as well as stacks. With the aid of PCB technology, current density distribution could be achieved by measuring the local current density inside the fuel cell rather than measuring the integrated current from the whole area of the fuel cells, which would be more precisely reflecting the local electrochemical reaction. Thus, using the segmented cell will know about the distribution of current density more precisely and allow for a correct interpretation of measured current density distributions (4).

German Aerospace Center (DLR) has applied this technique with segmented bipolar plates to study locally resolved current densities in single cells or stacks and used it in various fuel cell types, like PEMFCs, direct methanol fuel cells (DMFCs), and solid oxide fuel cells (SOFCs). One aim of these studies is to identify regular cell operation without disturbances and errors $(5,6)$. 
However, the reliability of fuel cell is one of the important issues for the successful commercialization of fuel cell, which is extremely important for the high-temperature PEM fuel cell. The current density distribution measurement with PCB is also developed to detect early disturbances and malfunctions of the cell and to identify their influence on accelerated degradation. Through the study of the evolution of current density distributions, an evolving membrane leakage is identified by characteristic local behavior. The spreading of the leakage can be followed and is of interest to evaluate the global significance for stack operation. The causes of cell degradation and the understanding of fundamental mechanisms of cell degradation can be investigated. Thereby a way to avoid the membrane leakage formation and to extend the life of fuel cell may be found and, finally catastrophic failure of the whole stack may be avoided.

\section{Experimental}

\section{Principle of segmented bipolar plate}

A bipolar plate of a cell generally conducts current in three dimensions. To measure the current density distribution in cells with plane MEAs, the bipolar plate is replaced by a measurement board which conducts current in only one dimension, i.e. only in the through plane direction. This is achieved by using a plate made of non-conducting material which is covered with conducting layers. The top and back layer of the board are then connected which each other by conducting wires and the layer which is in contact with the MEA (i.e. segmented surface) is divided into segments to avoid lateral (i.e. inplane) conduction (Figure 1). This setup forces the current between segmented surface and collector surface (back layer) on discrete pathways which connect every segment surface with the collector surface. The current flow in each of the pathways may then easily be measured by integrating calibrated resistors in each path and measure the voltage drop across each resistor via sense wires connected to a voltmeter.

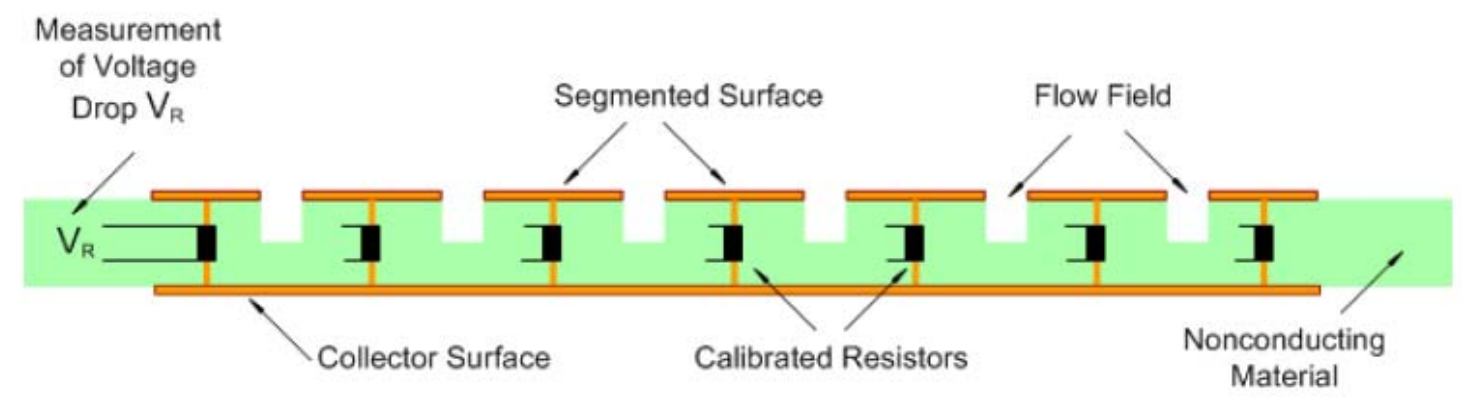

Figure 1. Schematic drawing of segmented bipolar plates 


\section{Design of segmented bipolar plates}

Most in-situ methods are integral and therefore do not allow a locally resolved analysis of cells. It is important as inhomogeneous distribution of reactants and products often occur depending on the operating conditions. Locally resolved current density measurements in single cells have been developed. A segmented bipolar plate based on printed circuit board (PCB) technology with integrated temperature sensors was used in the single cell to analysis the locally resolved current density distributions. A segmented bipolar plate with 49 segments for the single cell was used in this experiment. The schematic of flow field for the PCB is shown in Figure 2. The flow field channels were machined directly into the plate. G1 is the inlet part, A1 is the outlet part. In the single cell, the PCB with segmented current collector and flow field was installed into the anode side for the current density distribution measurements also as error detection techniques.

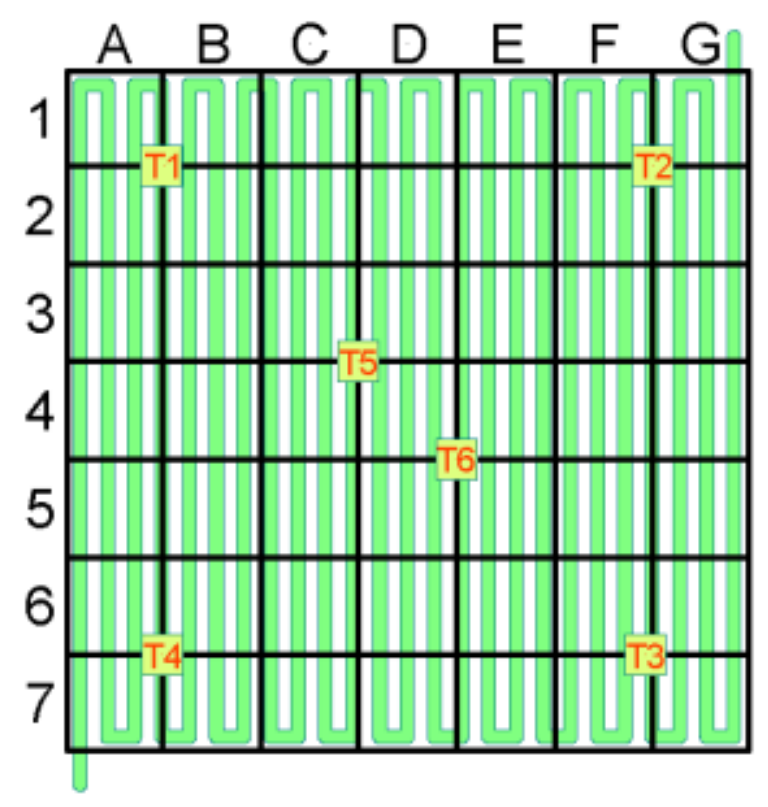

Figure 2. The scheme of the PCB for current density distributions

With the aid of segmented cell technology, the operating conditions (such the humidity of the cell) as well as the fuel cell components, especially the flow fields and electrode / electrolyte interface structures can be optimized. In addition, the current density distribution measurements have a high potential for the application of controlling fuel cell systems. Furthermore, early detection of malfunctions, errors and failures in fuel cell can be implemented.

\section{$\underline{\text { Test procedure and operating conditions }}$}

The humidification temperatures of anode and cathode were set. The cell was tested for gas leakage and cross-over before actual testing. Hydrogen stoichiometry $\left(\lambda \mathrm{H}_{2}\right)$ and air 
stoichiometry ( $\lambda$ air) were kept constant, respectively, at 1.5 and 2 . During the current density measurement, the segments information was recorded by the data capture. The measurement setup worked independently from the electric load unit and the control system of the fuel cell.

\section{Results and Discussion}

\section{$\underline{\text { Segmented and global current density distribution }}$}

Figure 3 shows the plots of global cell current density and voltage versus time as well as locally resolved currents for operation of the fuel cell at $120^{\circ} \mathrm{C}$. The humidification temperature of anode and cathode were also set at $120^{\circ} \mathrm{C}$. From the plots of global and open circuit voltage versus time (Figure 3a), it was found that at potentiostatic operation condition, the global current density gradually decreased to zero after 4:40 h. It is observed that after the current density dropped to zero, the setting voltage started to decrease with time. In Figure 3b, the time dependence of the segments E1, G6, F1, D1, G5, and G4 are displayed. It was found that the current density of the segments decreased from positive values to zero and then even exhibited negative current values. The negative current densities can reach exceptionally high values in some segments $\left(-6000 \mathrm{~mA} / \mathrm{cm}^{2}\right)$, in particular G4 and G5 segments. A negative current density first appeared in the E1 segment, followed by G6, F1, D1, G5, and G4 segments in this sequence. In earlier investigations, it was found that in case of membrane rupture negative current densities appeared in the vicinity of the hole. Therefore the observations here are also interpreted as indicative of a spreading membrane leakage: the leakage first started at the E1 segment, and then another leakage appeared in G6 following the appearance of negative currents. Furthermore, leakages in F1, D1, G5, and G4 segments gradually appeared, which were all located in the boundary of the flow field and gasket. It could be found that the membrane fissures mostly happened at the edges where the membrane is clamped. After the first hole formed, the inhomogeneous current density distribution exacerbated and led to severe mechanical strain. Finally, the cell voltage decreased gradually due to massive gas crossover and mixed potential formation on both sides. 


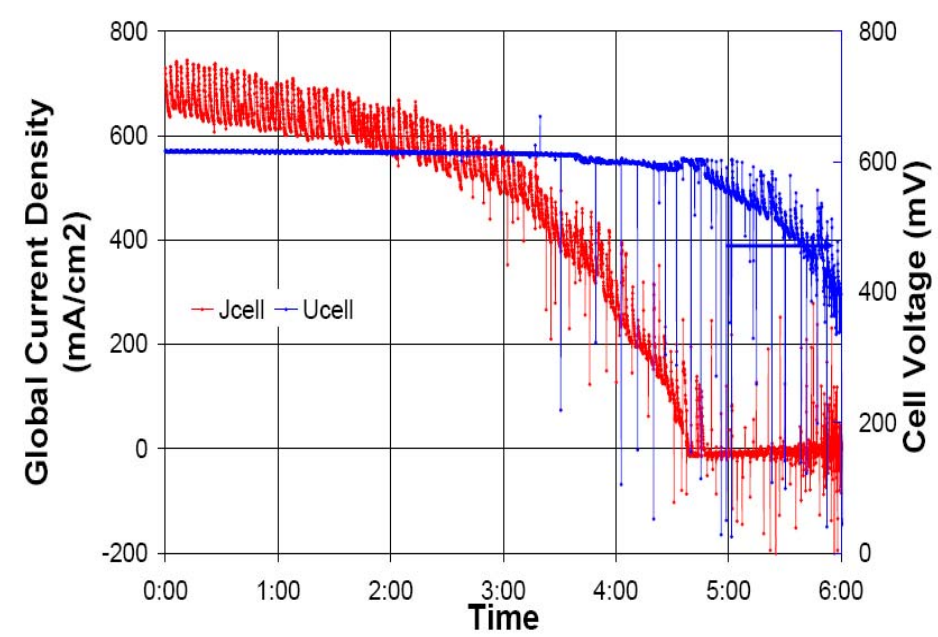

a,

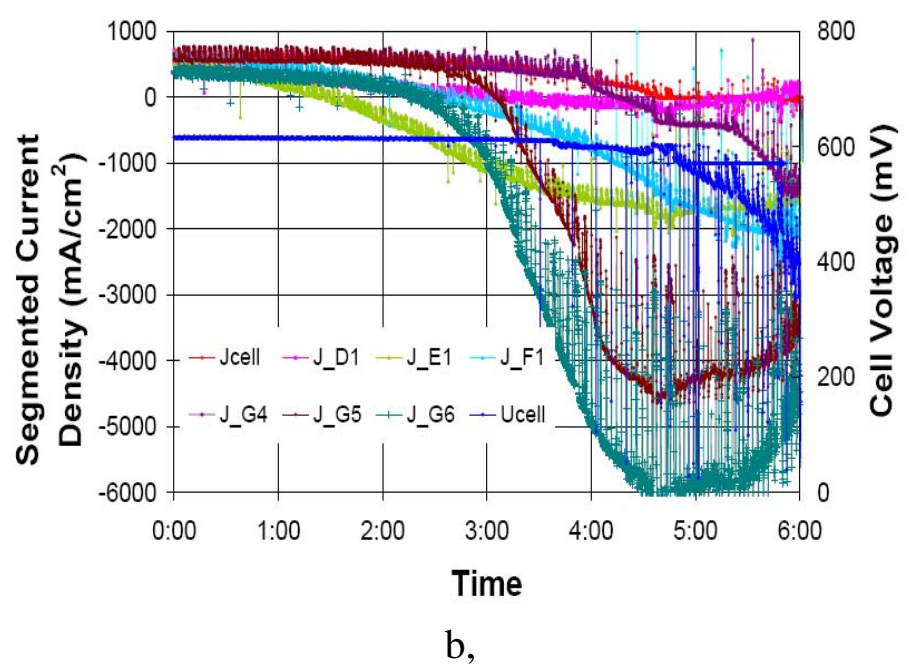

Figure 3. Plots of segmented and global current density distribution and open circuit voltage versus time, a, global and open circuit voltage; b segmented cell (E1, G6, F1, D1, G5 and G4).

\section{Mapping of current density distributions during the evolution of membrane leakage}

Figure 4 shows a map of current density distribution of the cell at the start of the failure and during evolution of the malfunction. It could be also found that a negative current density first appeared in the area of segment (E1) (the value of negative current is indicated by the legend, $-2400 \sim 0 \mathrm{~mA} / \mathrm{cm}^{2}$ ). It further identified that a leakage of membrane started in the area of E1 segment. After some time, another leakage appeared in G6, which is near the boarder of the flow field. Furthermore, more and more leakages (G6, F1, D1, G5, and G4) appear which were all located in the boundary of the flow field and gasket. Then, the leakage affects the neighbor regions and spreads to the middle of the membrane and affects an increasing part of the active area. Finally, the membrane is totally destroyed, which is evident from the current decreased to zero and also a decrease of voltage. 

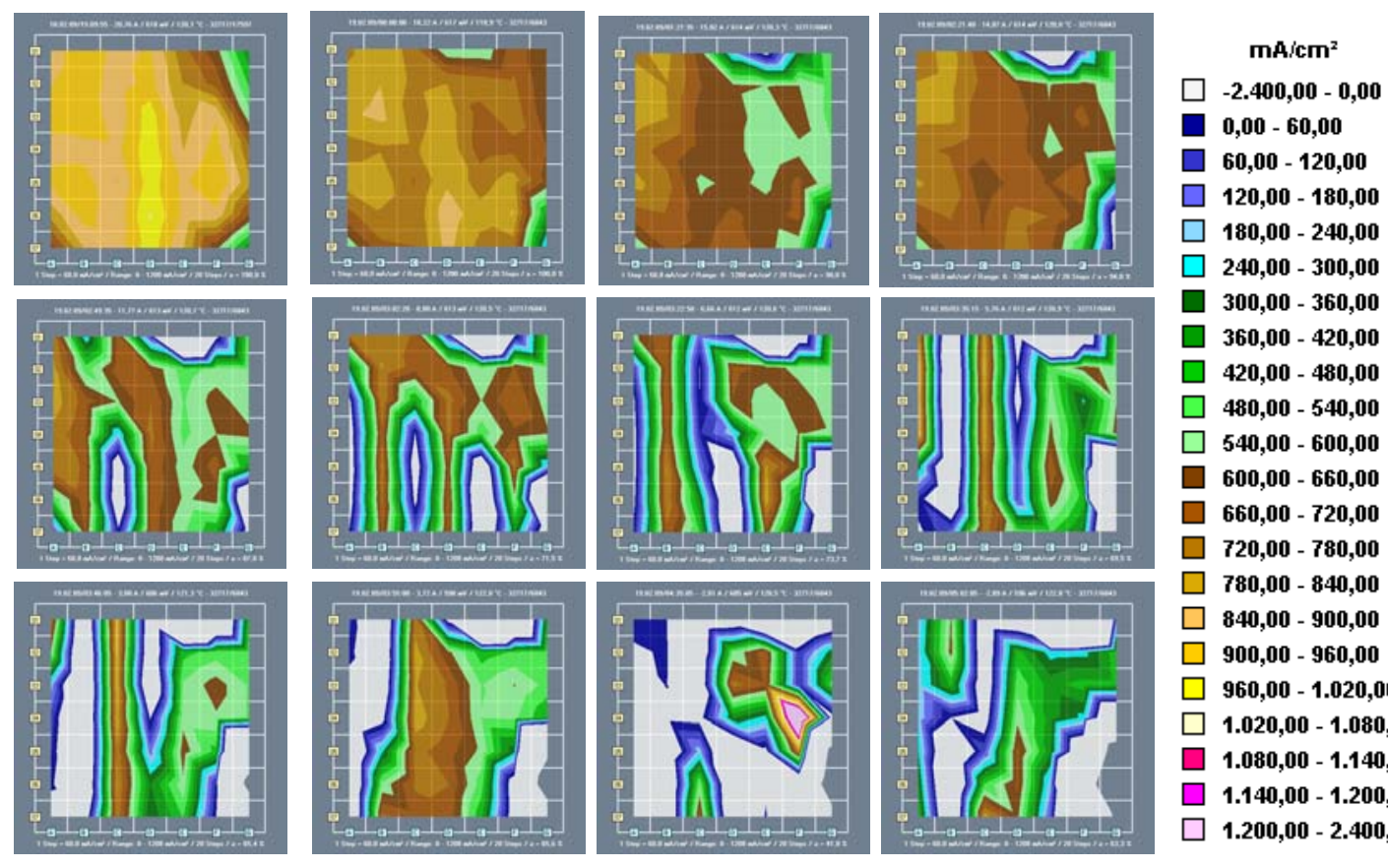

$300,00-360,00$

$360,00-420,00$

$\square \mathbf{4 2 0 , 0 0}-\mathbf{4 8 0 , 0 0}$

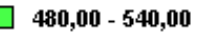

$540,00-600,00$

$600,00-660,00$

$660,00-720,00$

$720,00-780,00$

$780,00-840,00$

840,00 - 900,00

$900,00-960,00$

$960,00-1.020,00$

$1.020,00-1.080,00$

$1.080,00-1.140,00$

$1.140,00-1.200,00$

$1.200,00-2.400,00$

Figure 4. Mapping of current density distributions during the evolution of membrane leakage. (absolute values of current density plotted)

\section{Evolution of operation conditions during the evolution of membrane leakage}

Figure 5 is the display of the evolution of air and hydrogen pressure, the global current, and the segmented cell temperature two days ago before the membrane leakage started. The temperature of the segments reflected precisely the real temperature of the MEA due to its close vicinity to the membrane. The accelerated cell degradation was evident in the time dependence of these values. It was found that the pressure of air and hydrogen rapidly decreased and at the same time the temperature steeply decreased from $120^{\circ} \mathrm{C}$ to $112^{\circ} \mathrm{C}$ at very short time (85 seconds). Also, the current diminished very quickly. The sudden drop of the hydrogen and air pressure caused the evaporation of the local water, which resulted to the large variation of local water content. It also induced the fast cooling of the local membrane and big temperature gradient for different local membranes. The temperature of membrane is not equally distributed. Some local part of the membrane was at $120^{\circ} \mathrm{C}$, while other areas experienced lower temperatures. As a consequence the membrane will swell or shrink in different areas leading to mechanical strain due to the inhomogeneous water content and the temperature gradient. From these results, it was found that the inhomogeneous water distribution and temperature gradient in the MEA caused the inhomogeneous current density distributions (7), and induced significant mechanical stresses of the membrane. Thus, the inhomogeneous current density distribution and mechanical strain and stress on MEA were exacerbated leading to the future fissures and tearing of the membrane near the edges of the cell holder. 


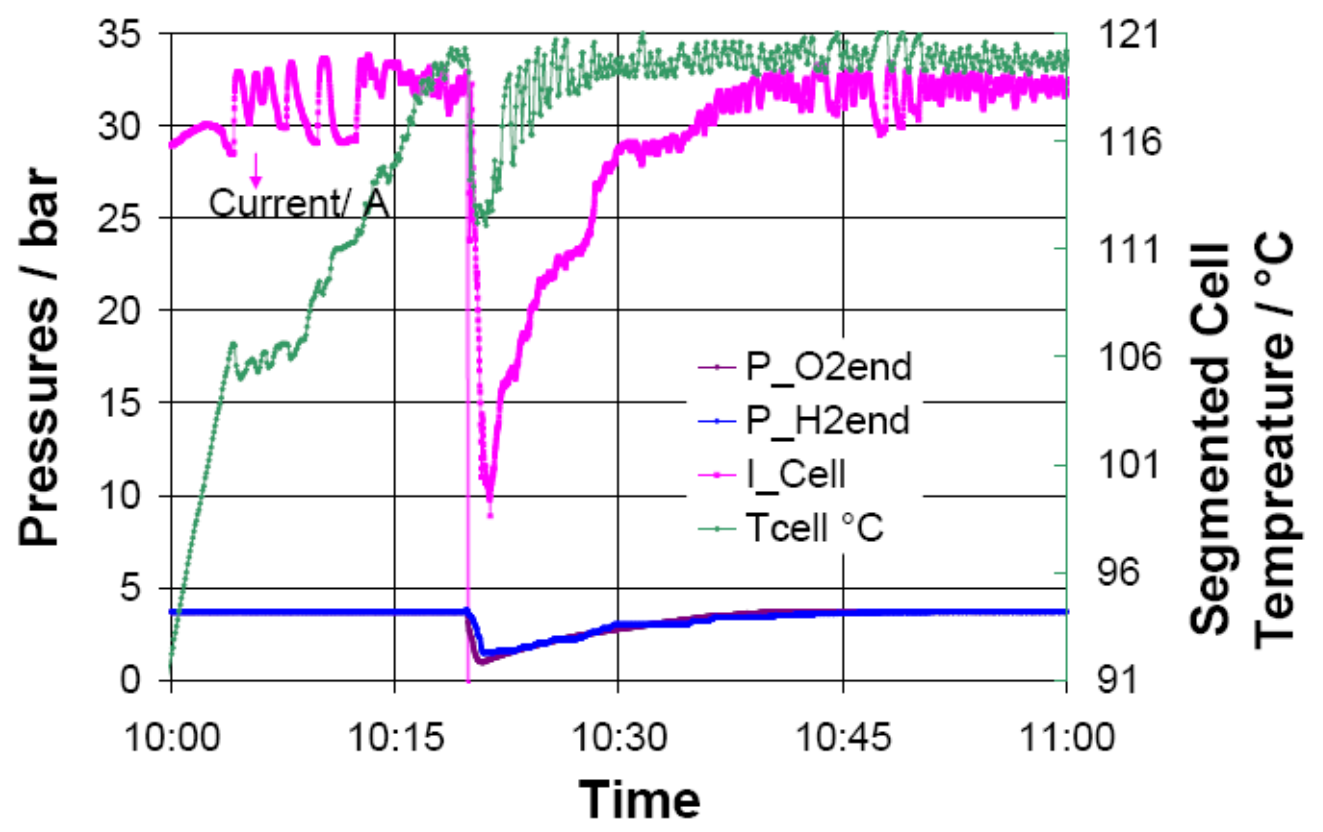

Figure 5. Evolution of pressure of air and hydrogen, current, and the segmented cell temperature two days ago before the membrane leakage started.

\section{Summary}

With the aid of segmented cell technology, the evolution of local current density distributions, starting locations of membrane leakages and their spreading could be detected. The steeply decreased pressure and consequently the evaporation of water and the fast cooling of the local membrane was the main reason for the degradation of the membrane. It caused large temperature gradient in different local membranes, which resulted in inhomogeneous current density distribution and mechanical strain and stress. All lead to fissures and tearing of the membrane near the edges of the cell holder. Through the study of the evolution of leakages in the fuel cell, malfunction could be detected at an early stage and thereby catastrophic failure of the whole stack may be avoided. Thus the durability of the fuel cell system may be extended by identifying early malfunctions and taking counter measures.

\section{Acknowledgments}

The authors gratefully acknowledge the financial support from German Aerospace Center (DLR). Dr. Rui Lin also thanks the doctoral program of higher education (No.1700279016), program for young excellent talents in Tongji University (No. 2006KJ022). 


\section{References}

1. M. Schulze, ECS Transcations, 12, 177 (2008).

2. D. G. Strickland, S. Litster and J. G. Santiago, J. Power Sources, 174, 272 (2007).

3. R. Eckl, R. Grinzinger and W. Lehnert, J. Power Sources, 154, 171 (2006).

4. M. Schulze, E Gülzow, St, Schönbauer, T Knöri and R. Reissner, J. Power Sources, 173, 19 (2007).

5. M. Schulze, E. Gulzow, K. A. Friedrich, P. Metzger and G. Schiller, ECS Transactions, 5, 49 (2007).

6. C. Wieser, A. Helmbold, E. Gulzow, J. Appl. Electrochem., 30, 803 (2000).

7. D.J.L. Brett, S. Atkins, N. P. Brandon, N. Vasileiadis, V. Vesovic and A.R. Kucernak, J. Power Sources, 172, 2 (2007). 